\title{
Diffracting a Beam of Organic Molecules
}

\author{
Researchers create diffraction patterns using beams made of large organic \\ molecules, a first step toward creating an interferometer for these \\ systems.
}

By Sophia Chen

$\Lambda$ ccording to quantum mechanics, all matter can exhibit wave-like phenomena, such as interference and diffraction. Physicists have long observed diffraction and its resulting patterns using electrons and atoms. But making the patterns for larger objects is more challenging because their ill-defined energy structures and complex thermal vibrations introduce pattern-destroying noise. Now, Christian Brand, at the University of Vienna, Austria, and colleagues have demonstrated Bragg diffraction using large organic molecules-a first step to creating a molecular interferometer.

In Bragg diffraction, particles travel through a grating, which alters their momenta such that the particles exit the grating at two angles. A typical interferometer uses this effect to divide every particle's wave function into two wavelets that travel along separate paths before interfering. To enable more precise interferometry measurements, researchers try to maximize the

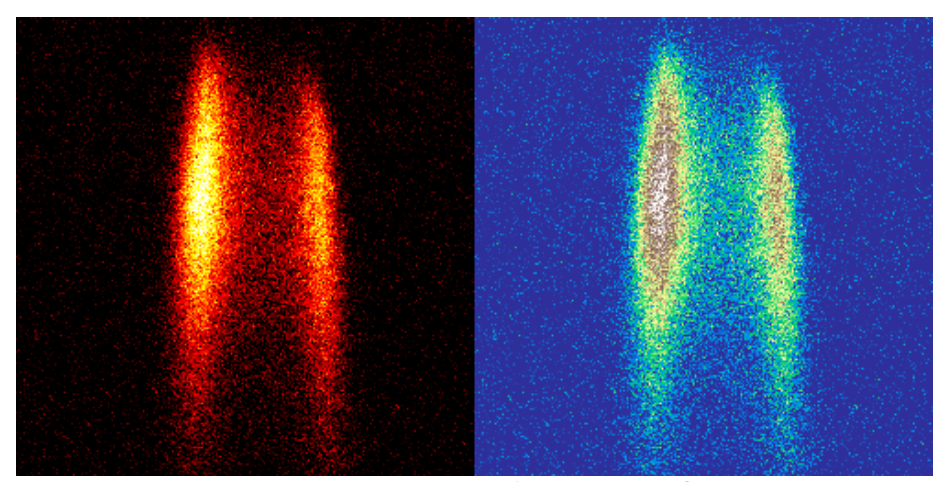

Credit: Quantum Nanophysics Group/University of Vienna momentum difference between the two paths.

The researchers created their Bragg grating using standing light waves generated by retroreflecting a green laser from a mirror. In two separate experiments, they sent beams of the antibiotic ciprofloxacin, a 41-atom polar molecule, and the dye phthalocyanine, a nonpolar 58-atom molecule, through this grating. Both experiments produced diffraction patterns with two peaks, corresponding to groups of molecules with a large momentum difference between them.

Despite the molecules' complex structures and vibrations, the team found that they could replicate the diffraction patterns using a model that treated the molecules as polarizable point particles. As phthalocyanine formed the pattern-despite absorbing light of the wavelength of the laser-the researchers say that this setup could function as a beam splitter for a range of organic molecules. But first, the researchers plan to use their Bragg diffraction patterns to make precise measurements of the two molecules' properties.

This research is published in Physical Review Letters.

Correction (17 July 2020): The title was updated to correct the misspelling of "Diffracting."

Sophia Chen is a freelance science writer based in Columbus, Ohio. 